\title{
Effekte eines multimodularen ambulanten Trainingsprogramms für Patienten mit COPD ${ }^{1}$
}

\section{Benefits of a Multimodular Outpatient Training Program for Patients with COPD}

Autoren

Institute
O. Göhl ${ }^{1}$, H. Linz ${ }^{2}$, T. Schönleben ${ }^{1}$, B. Otte ${ }^{1}$, J. Weineck ${ }^{3}$, H. Worth

Klinikum Fürth, Medizinische Klinik I

2 Therapie im Park, Praxis für Physiotherapie

Institut für Sport und Sportwissenschaft der Universität Erlangen-Nürnberg eingereicht 1.11.2005

akzeptiert 18.5.2006

Bibliografie

DOI $10.1055 / \mathrm{s}-2006-944235$

Pneumologie 2006; 60; 529-536

(c) Georg Thieme Verlag KG

Stuttgart · New York

ISSN 0934-8387

Korrespondenzadresse

Dr. phil. Oliver Göhl

Klinikum Fürth

Medizinische Klinik I

Jakob-Henle-Str. 1

90766 Fürth

oliver.goeh|@klinikum-fuerth.de

\section{Zusammenfassung}

Hintergrund: Die qualitative und quantitative Zusammensetzung sowie die chronologische Reihenfolge verschiedener Trainingsinhalte und -methoden für ein effektives Training von Patienten mit COPD sind noch nicht endgültig geklärt. Wir haben die Kombination verschiedener effektiver Komponenten der pneumologischen Rehabilitation in den Umsetzungsmöglichkeiten Lungensportgruppe, Heimtraining und Training in einer Gesundheits- und Fitnesseinrichtung untersucht. Methode: Die Patienten wurden randomisiert einem 12-monatigen Trainingsprogramm zugeführt oder erhielten kein Training.

Patienten und Ergebnisse: Die Trainingsgruppe (TG) $\left(n=10\right.$, Alter 62,5 Jahre, $\mathrm{FEV}_{1}: 53,4 \%$ Soll $)$ erreichte eine durchschnittliche Zunahme der maximalen symptomlimitierten Sauerstoffaufnahme um $10 \%(p=0,01)$ von $1,30 \pm 0,41 \mathrm{~L} /$ Minute auf $1,44 \pm 0,38 \mathrm{~L} /$ Minute, der maximalen Belastbarkeit um $14 \%(p=0,007)$ von $82 \pm 39$ Watt auf $93 \pm$ 43 Watt und der Sechs-Minuten-Gehstrecke um 79 Meter $(p=0,003)$. In der Kontrollgruppe (KG) $\left(\mathrm{n}=9\right.$, Alter 63, 2 Jahre, $\mathrm{FEV}_{1}: 53,7 \%$ Soll) wurden keine signifikanten Veränderungen beobachtet. Die mittels SF36 und SGRQ ermittelte Lebensqualität nahm in der TG nicht, jedoch in der KG signifikant zu.

Schlussfolgerungen: Dieses ambulante Trainingsprogramm ist effektiv und für eine längerfristige Nachsorge von Patienten mit COPD geeignet.

\section{Abstract}

Background: The partition and chronological sequence of the different training items and modalities necessary for an effective training of patients with chronic obstructive pulmonary disease (COPD) remains a subject of debate. We investigated the combination of effective components of pulmonary rehabilitation in the settings outpatient lung sports group, home-based training and training in a gym.

Methods: Subjects were randomized to a 12-month training program and a no training group.

Patients and Main Results: The training group $\left(n=10\right.$, mean age: 62,5 years, $\mathrm{FEV}_{1} 53,4 \%$ predicted) reached an average increase in peakVO of $10 \%(p=0,01)$ from $1,30 \pm 0,41 \mathrm{~L} /$ minute to $1, .44 \pm 0,38 \mathrm{~L} /$ minute, in peak work rate of $14 \%$ $(\mathrm{p}=0,007)$ from $82 \pm 39 \mathrm{~W}$ to $93 \pm 43 \mathrm{~W}$ and in the six-minute walking distance of 79 meters $(p=0,003)$, whereas in the control group $(n=9$, mean age: 63,2 years, $\mathrm{FEV}_{1} 53,7 \%$ predicted) no significant changes were observed. The quality of life obtained by use of the SF36-questionnaire and SGRQ also significantly improved in the training group, but not in the control group.

Conclusion: This outpatient rehabilitation program is effective and appropriate for long-term rehabilitation of patients with COPD.

\section{Einleitung}

Die pneumologische Rehabilitation hat sich als wichtige evidenzbasierte Maßnahme im Management der COPD etabliert [1]. Körperliches Training wird als zentraler Bestandteil [2] eingestuft. Effekte werden u.a. in den Bereichen Leis-
${ }^{1}$ Diese Arbeit wurde als freier Vortrag unter dem Titel „Effekte eines ambulanten, dynamischen körperlichen Trainingsprogramms für Patienten mit COPD“ auf dem Kongress der Deutschen Gesellschaft für Pneumologie in Frankfurt 2004 präsentiert. 
tungsfähigkeit und Lebensqualität erreicht, die Umsetzung kann stationär, ambulant oder als Heimtraining erfolgen. Die eingesetzten Trainingskonzeptionen sind höchst unterschiedlich in Bezug auf Trainingsintensität, -dichte, -dauer, -umfang, -häufigkeit, -inhalte und -methoden [3]. Der Stellenwert einzelner Trainingsinhalte (qualitativer Aspekt) wird zunehmend deutlicher, der Aspekt der quantitativen Relation und die zeitliche Reihenfolge verschiedener Trainingsinhalte und -modalitäten im Rahmen längerfristiger Interventionen sind bisher noch unzureichend untersucht.

Vor diesem Hintergrund haben wir verschiedene Umsetzungsmöglichkeiten („Lungensportgruppe“, „Heimtraining“ und „Training in einer Gesundheits- und Fitnesseinrichtung“) im Rahmen einer 12-monatigen Rehabilitationsmaßnahme kombiniert. Vorgestellt wird ein trainingswissenschaftlich orientiertes Modell, in dem bewährte Inhalte in einer bestimmten zeitlichen Reihenfolge unter Berücksichtigung des jahreszeitlichen Verlaufs und der Ausgangssituation des Patienten (Dekonditionierung) angeordnet wurden. Die zentrale Frage ist: Führt dieses komplexe längerfristige Trainingsprogramm für Patienten mit mittel- und schwergradiger COPD zu einer Verbesserung der Bereiche Leistungsfähigkeit und Lebensqualität?

\section{Material und Methoden}

Für die Rekrutierung wurden niedergelassene Pneumologen und relevante Hausärzte der Region angeschrieben oder telefonisch angefragt. Ferner wurde ein Patientenforum mit Bewerbung über die lokale Presse durchgeführt. Durch diese Maßnahmen konnten 55 Patienten gewonnen werden. Nach Überprüfung der Ein- und Ausschlusskriterien und Einverständniserklärung wurden aus den verbleibenden Patienten 34 Teilnehmer per Losverfahren in die Studie aufgenommen. Alle Patienten waren während der Intervention weiterhin in fachärztlicher Betreuung. Die eingeschlossenen Patienten hatten eine mittel- bis schwergradige COPD [4] und waren zwischen 50 und 75 Jahren alt. Die Ausschlusskriterien beinhalteten symptomatische oder dekompensierte koronare Herzkrankheit, hämodynamisch wirksame Herzrhythmusstörungen oder Kartitiden, unzureichend eingestellte arterielle Hypertonie, respiratorische Globalinsuffizienz, bedeutsame respiratorische Partialinsuffizienz $\left(\mathrm{paO}_{2}\right.$ $<50 \mathrm{~mm} \mathrm{Hg}$, bzw. $\mathrm{SaO}_{2}<80 \%$ in Ruhe), Rechtsherzbelastung bei pulmonaler Hypertonie in Ruhe (accelerative time $>100 \mathrm{msec}$ ), positiver Bronchodilatationstest mit einer $\mathrm{FEV}_{1}-\mathrm{Zunahme}_{\mathrm{H}} \mathrm{15} \%$ gegenüber dem Ausgangswert nach Inhalation eines kurzwirksamen Beta-2-Sympathomimetikums, exazerbierte COPD, starkes Übergewicht $(\mathrm{BMI}>35)$ und eingeschränkte Belastbarkeit auf dem Fahrradergometer (<50 Watt).
Von ursprünglich je 17 Teilnehmern konnten in der Trainingsgruppe $n=10$ und in der Kontrollgruppe $n=9$ Personen ausgewertet werden ( Tab.1). Folgende 7 Ausfälle waren in der Trainingsgruppe zu verzeichnen: periphere arterielle Verschlusskrankheit (2), Schulterbruch (1), Prostatakrebs (1), Infektexazerbation in Haupttrainingsphase (1) und Infektexazerbation im Zeitraum der Abschlussuntersuchungen (2). Aufgrund von Todesfall (2), periphere arterielle Verschlusskrankheit (2), Infektexazerbation im Zeitraum der Abschlussuntersuchungen (2) und Non-Compliance (2) konnten 8 Fälle in der Kontrollgruppe nicht ausgewertet werden.

\section{Studiendesign}

34 Personen wurden randomisiert (Losentscheid) einer Trainings- und einer Kontrollgruppe zugeführt. Die Trainingsgruppe erhielt ein komplexes Trainingsprogramm, die Kontrollgruppe erhielt keine Therapie. Beide Gruppen erhielten eine vergleichbare leitliniengemäße Medikation. Die Personen durchliefen eine Testbatterie vor und nach 12 Monaten Intervention. Aus Gründen der Trainingssteuerung erhielt die Trainingsgruppe weitere Spiroergometrieuntersuchungen nach sechs und neun Monaten. Die Studie ist als prospektive, randomisierte KontrollStudie zu werten.

\section{Terminologie und Systematik im Rahmen der quantitativen Darstellung}

Im Rahmen dieser Intervention wurde die in - Tab. 2 dargestellte Systematik und Terminologie gewählt. Darauf basierend wurden alle Inhalte in eine prozentuale Relation gebracht ( Abb. 1). Hierfür wurden für jede Trainingseinheit die zeitlichen Umfänge der einzelnen Trainingskomponenten gerundet aufsummiert und auf die einzelnen Trainingsphasen und Trainingsorte umgerechnet.

\section{Umsetzung des Trainingsprogramms}

Zur Umsetzung der Trainingskonzeption (November 2000-Oktober 2001) wurden wohnortnahe Umsetzungsmöglichkeiten (Lungensportgruppe, Heimtraining und Gesundheits- und Fitnesseinrichtung) unter Ausnutzung der spezifischen Vorteile kombiniert. In der Gesundheits- und Fitnesseinrichtung wurden Krafttrainingsgeräte genutzt. Die notwendigen Trainingsumfänge im Ausdauerbereich zum Erreichen eines Trainingseffektes wurden über das Heimtraining, hauptsächlich durch Fahrrader-

\begin{tabular}{|c|c|c|c|}
\hline & Trainingsgruppe $(n=10)$ & Kontrollgruppe (n=9) & \multirow{8}{*}{$\begin{array}{l}\text { Tab. } 1 \text { Charakteristik der Pa- } \\
\text { tienten zu Beginn der Interven- } \\
\text { tion }\end{array}$} \\
\hline männlich:weiblich & $6: 4$ & $7: 2$ & \\
\hline Alter (Jahre) & $62,5 \pm 7$ & $63,2 \pm 8,5$ & \\
\hline Größe (cm) & $166,3 \pm 9,1$ & $169,9 \pm 6,9$ & \\
\hline Gewicht (kg) & $77,7 \pm 20,3$ & $75,1 \pm 14,8$ & \\
\hline $\mathrm{FEV}_{1}(\%$ Soll $)$ & $53,4 \pm 10,7$ & $53,7 \pm 5,8$ & \\
\hline $\mathrm{VO} 2_{\text {peak }}(\mathrm{L} / \mathrm{min})$ & $1,3 \pm 0,4$ & $1,4 \pm 0,5$ & \\
\hline $6 \mathrm{MWD}(\mathrm{m})$ & $415 \pm 89,7$ & $456 \pm 83$ & \\
\hline
\end{tabular}




\begin{tabular}{|c|c|c|c|c|c|}
\hline & $\begin{array}{c}\text { Schulung/ } \\
\text { Theorie }\end{array}$ & Kraft & Ausdauer & $\begin{array}{c}\text { Beweglich- } \\
\text { keit }\end{array}$ & Koordination \\
\hline $\begin{array}{c}\text { Phase 4: Anaerobe } \\
\text { Trainingsphase (3 Monate) }\end{array}$ & $6 \%$ & $6 \%$ & $74 \%$ & $9 \%$ & $5 \%$ \\
\hline $\begin{array}{c}\text { Phase 3: Aerobe } \\
\text { Trainingsphase (3 Monate) }\end{array}$ & $8 \%$ & $15 \%$ & $65 \%$ & $8 \%$ & $4 \%$ \\
\hline $\begin{array}{c}\text { Phase 2: Anpassungsphase } \\
\text { (3 Monate) }\end{array}$ & $7 \%$ & $62 \%$ & $9 \%$ & $15 \%$ & $7 \%$ \\
\hline $\begin{array}{c}\text { Phase 1: Gewöhnungsphase } \\
\text { (3 Monate) }\end{array}$ & $17 \%$ & $39 \%$ & $3 \%$ & $24 \%$ & $17 \%$ \\
\hline
\end{tabular}

Abb.1 Quantitative Relation der verschiedenen Trainingskomponenten während der 12-monatigen Intervention. Für diese prozentuale Darstellung wurden die Trainingsvolumina aller Trainingsinhalte (gemäß Tab. 1) jeder Trainingseinheit gerundet aufsummiert und für die vier Trainingsphasen dargestellt.

\begin{tabular}{|c|c|}
\hline Komponente & Inhalt (Auswahl) \\
\hline Kraft & $\begin{array}{l}\text { Übungen mit freien Gewichten, Theraband oder Übungen an Krafttrainingsgeräten, } \\
\text { Übungen ohne Gerät in verschiedenen Modalitäten (z. B. dynamisch, isometrisch) }\end{array}$ \\
\hline Ausdauer & Gehen, Walking, Fahrradergometer, Treppensteigen, Stepper \\
\hline Beweglichkeit & $\begin{array}{l}\text { Allgemein: Stretching, Mobilisation } \\
\text { Speziell: Elemente der Physiotherapie wie Drehdehnlagen }\end{array}$ \\
\hline Koordination & $\begin{array}{l}\text { Allgemein: Aktivitäten des täglichen Lebens (ADL), Übungen zur Koordination mit } \\
\text { oder ohne Gerät, Spiele und Übungen mit koordinativen Aspekten } \\
\text { speziell: Atemtechniken, atemerleichternde Ausgangsstellungen, Entspannungs- } \\
\text { übungen }\end{array}$ \\
\hline Schulung/Theorie & $\begin{array}{l}\text { Allgemein: Erklärungen und Anweisungen für die verschiedenen Übungen und Tech- } \\
\text { niken wie Walking, Kraftübungen, Dehnübungen und allgemeiner Schulung. } \\
\text { Speziell: Elemente der Patientenschulung, theoretische Aspekte z. B. der dynami- } \\
\text { schen Überblähung oder Aspekten zu Inhalten der speziellen Koordination. }\end{array}$ \\
\hline
\end{tabular}

\section{Makrozyklus}

Gewöhnungsphase

November-Januar

Anpassungsphase

Februar-April

Aerobe Trainingsphase

Mai-Juli

Anaerobe Trainingsphase

August-Oktober

\section{durchschnittliche Trainingszeit} pro Woche [Stunden/Woche]

2,4

2,4

3,6

4,2 gerundete prozentuale Trainingszeit in den einzelnen Settings

$60 \%$ Lungensportgruppe

$15 \%$ Heimtraining

$25 \%$ Fitness- und Gesundheitseinrichtung

$60 \%$ Lungensportgruppe

$40 \%$ Fitness- und Gesundheitseinrichtung

$40 \%$ Lungensportgruppe

$60 \%$ Heimtraining

$35 \%$ Lungensportgruppe

$65 \%$ Heimtraining
Tab. 2 Terminologie und Systematik der Intervention gometertraining, abgedeckt. Die Lungensportgruppe stellte das konzepttragende Element dar. Die ursprünglich 17 Teilnehmer trafen sich wöchentlich für 90 Minuten in einer Turnhalle, um mit einem Fachübungsleiter Kurseinheiten durchzuführen. Alle relevanten theoretischen und praktischen Inhalte wurden dort vermittelt. Für alle Kurseinheiten gab es exakte schriftliche Ausarbeitungen (Stundenbilder). Darin enthalten waren neben den konkreten Inhalten und zeitlichen Vorgaben auch Aufstellungsund Ordnungsformen sowie Hinweise auf methodisches und didaktisches Vorgehen. Zur Koordination des Trainings wurden individuelle Wochentrainingspläne für das Heimtraining und die Einheiten in der Gesundheits- und Fitnesseinrichtung ausgegeben. Alle Trainingseinheiten (Kursstunden, Krafttraining und Heimtraining) wurden mit einem speicherfähigen Herzfrequenzmesser (Polar Accurex, Polar Electro, Kemple, Finnland) aufgezeichnet. Die Daten wurden zu Beginn der Kursstunden in den PC eingelesen und gaben u.a. Auskunft über Herzfrequenzverhalten, Tag, Uhrzeit und Dauer der Trainingseinheiten. Relevante Einheiten wurden ausgedruckt, bewertet und den Teilnehmern wieder ausgehändigt.

\section{Trainingsprogramm}

Einleitend ein Überblick über den modularen Aufbau der Trainingskonzeption ( Tab. 3 und Abb. 1). Die Inhalte der Kursstunden unterschieden sich von denen des Heimtrainings und denen in der Gesundheits- und Fitnesseinrichtung.

In Phase 1 (Woche 1-12) wurden grundlegende Techniken, u.a. Atemtechniken, atemerleichternde Ausgangsstellungen, Drehund Dehnlagen, Aktivitäten des täglichen Lebens, allgemeine Dehntechniken, Selbstwahrnehmung und Bewegungsausführungen im Krafttraining vermittelt sowie Spiel- und Übungsformen mit und ohne Gerät durchgeführt. Die Einheiten besaßen Übungscharakter und stellten eher eine Lernphase der Techniken und Bewegungsabläufe als ein Training im eigentlichen Sinne dar.

Die Umsetzung des Krafttrainings ( Tab. 4) in der Gesundheitsund Fitnesseinrichtung erfolgte in den Wochen 9-25 mit durchschnittlich einer betreuten Trainingseinheit pro Person und Trainingswoche. Eingesetzt wurden die Geräte Beinpresse, Latissimusstation, Bauchmaschine, Wadenheber und Schulterpresse. 


\begin{tabular}{|c|c|c|c|c|}
\hline $\begin{array}{l}\text { Woche der } \\
\text { Intervention }\end{array}$ & Inhalt & $\begin{array}{l}\text { Sätze, Wiederholungen } \\
\text { (RM) }\end{array}$ & Trainingshäufigkeit & Tab. 4 Krafttrainingsregime \\
\hline $9-10$ & $\begin{array}{l}\text { Einweisung } \\
\text { in die Geräte }\end{array}$ & - & $2 \times /$ Woche & \\
\hline $11-13$ & $\begin{array}{l}\text { Lern- und Gewöhnungsphase } \\
\text { Kraftausdauertraining }\end{array}$ & $2,15-20$ & $\begin{array}{l}1 \times, \text { von Woche } 12 \text { an } \\
2 \times / \text { Woche }\end{array}$ & \\
\hline $14-17$ & Kraftausdauertraining & 2 später 3,15 & $3 \times /$ Woche & \\
\hline $18-25$ & Querschnittstraining & 3,12 später 10 & $2 \times /$ Woche & \\
\hline
\end{tabular}

In den Pausen des Krafttrainings (ca. 2 Minuten) wurden u.a. Dehnübungen für die bearbeiteten Muskelgruppen durchgeführt. Großer Wert wurde auf eine saubere Bewegungsausführung, Kopplung der Bewegung an die Atmung, Erkennen und Vermeiden von Pressatmung und Durchführung der Dehnübungen gelegt.

In Phase 2 erfolgte der Übergang zum eigentlichen Training mit dem Ziel, vorgegebene Trainingsintensitäten einzuhalten. Inhaltlicher Schwerpunkt war das Krafttraining. Hierfür wurde im Anschluss an die Lernphase (Wochen 11-13) mit maximalen Wiederholungen (Wochen 14-25) gearbeitet. Die maximale Wiederholungsanzahl, die vor Ermüdung durchgeführt wird, verhindert das Beenden einer weiteren Wiederholung und stellt die Funktion der Gewichtsbelastung dar. Dies wird als „repetition maximum“ (RM) bezeichnet. Es spiegelt die Intensität der Belastung wieder. Entsprechend wird eine Gewichtslast, welche eine Ermüdung nach der 3. Wiederholung bewirkte als ein „three-repetition maximum“ (3-RM) bezeichnet. Bei gesunden Personen entspricht dies etwa $85 \%$ des Gewichtes, welches einmal bewegt werden kann.

Im Bereich Ausdauer wurden unterschiedliche Intensitäten anhand verschiedener „Bezugsgrößen“ Herzfrequenz, Atemfrequenz, Atemrhythmus oder auch Geschwindigkeit vorgegeben und jeweils Symptomatik sowie Borgskala (Grad der Atemnot und der körperlichen Belastung) erfragt und besprochen. Ziel war es, den Zusammenhang dieser Größen zu vertiefen sowie das Verhalten in unterschiedlich intensiven Bereichen zu erfassen. Speziell das Erkennen der dynamischen Überblähung im zeitlichen Verlauf der Belastung und adäquates Reagieren darauf wurde geübt. Der Einstieg erfolgte über Gehtraining mit Kurzzeitintervallen (2 Minuten Belastung, 2 Minuten Pause) welche systematisch auf Mittelzeitintervalle (20 Minuten) gesteigert wurden.

In Phase III (Mai, Juni, Juli) wurde ein Grundlagentraining auf dem Fahrradergometer durch die Dauermethode durchgeführt. Die Intensität lag bei ca. $40 \%$ der maximalen Wattzahl im Eingangstest und einer Herzfrequenz von ca. 76\% der maximalen Herzfrequenz im Eingangstest. Zum Vergleich: die anaerobe respiratorische Schwelle lag durchschnittlich bei $56 \%$ der maximalen Wattzahl und 83\% der maximalen Herzfrequenz. Gesteigert wurde generell die Trainingshäufigkeit (von 2 auf 4 Einheiten pro Woche) vor Trainingsdauer (von 25 auf 70 Minuten) vor Intensität (30 auf 55\% peak work rate). Die Teilnehmer wurden angewiesen, die auf den Spiroergometrieergebnissen beruhenden Herzfrequenzen und eine individuell optimale Atemfrequenz aufrecht zu erhalten. Bei Auftreten eines ermüdungsbedingten Herzfrequenzanstieges gegen Ende der Trainingseinheit wurde erlaubt, den Widerstand zu reduzieren. In den Wochen 30, 34, und 37 wurde Regenerationsphasen eingebaut: Trainiert wurde mit geringen Umfängen und Intensitäten.
In Phase IV wurde im Ausdauerbereich einmal pro Woche eine Grundlagenausdauereinheit über 60 Minuten durchgeführt. Die Intensität lag jetzt bei durchschnittlich $55 \%$ der maximalen Wattzahl im Eingangstest und einer Herzfrequenz von ca. 85\% der maximalen Herzfrequenz im Eingangstest. Hinzu kam ein später bis zu dreimal pro Woche durchgeführtes Intervalltraining mit einem Übergang von intensiven Mittelzeitintervallen (ab Woche $39 ; 4 \times 10$ später $3 \times 15$ Minuten) auf hochintensive Kurzzeitintervalle (ab Woche $43,6 \times 1$ später $10 \times 2$ Minuten). Die Intensität der Mittelzeitintervalle lag bei durchschnittlich 85\% der maximalen Wattzahl im Eingangstest und einer Herzfrequenz von ca. 90\% der maximalen Herzfrequenz im Eingangstest. Die Intensität der Kurzzeitintervalle lag bei durchschnittlich 100 - 120\% der maximalen Wattzahl im Eingangstest und einer Herzfrequenz von ca. $100 \%$ der maximalen Herzfrequenz im Eingangstest. Die bisher praktizierte Zyklisierung von 3 Wochen mit ansteigender Belastung zu 1 Woche Regeneration wurde beibehalten. Wie auch in Phase 3 wird durch die Regeneration die durchschnittliche wöchentliche Trainingszeit ( Tab.3) rein rechnerisch vermindert.

Gesamttrainingszeit dieser Intervention waren pro Person durchschnittlich 166 Stunden. Die Teilnahme in den 47 Kurseinheiten lag bei durchschnittlich $82 \%$.

\section{Untersuchungsverfahren}

\section{Belastungstests}

Spiroergometrie

Der maximale Belastungstest wurde auf einem elektromagnetisch gebremsten Fahrradergometer (ER 900, Erich Jaeger GmbH, Höchberg, Germany) durchgeführt. Ventilation und pulmonaler Gasaustausch wurden „breath by breath“ (Oxycon record, Jaeger, Höchberg, Germany) gemessen. Es wurden individuelle Testprotokolle erstellt, abhängig von Lungenfunktion, Referenzwerten [5] und einer Schätzung der maximalen Belastbarkeit der Patienten, mit dem Ziel, dass die Belastungsphase sich über einen Zeitraum von acht bis zwölf Minuten erstreckt. Die Belastung wurde kontinuierlich gesteigert (Rampenprotokoll). Die Testprotokolle blieben identisch für alle Untersuchungszeitpunkte und Patienten. Herzfrequenz, Sauerstoffsättigung und Blutdruck wurden durch EKG, Pulsoximetrie und sphygmomanometrisch registriert. Der Grad der körperlichen Anstrengung und Atemnot wurden durch die Borg-Skala ermittelt. In Ruhe, nach dem Leertreten, alle 2 Minuten, bei maximaler Belastung, sowie 2 und 5 Minuten nach dem Ende der Belastung wurde venöses Blut entnommen, um unmittelbar die Laktatwerte (Hitachi 717, Boehringer-Mannheim, Mannheim, Germany) zu bestimmen. In Ruhe und bei maximaler Belastung wurde kapilläres Blut aus dem Ohrläppchen entnommen, um den pH-Wert und die Partial- 


\begin{tabular}{|c|c|c|c|c|}
\hline & \multicolumn{2}{|l|}{ Trainingsgruppe } & \multicolumn{2}{|l|}{ Kontrollgruppe } \\
\hline & Prä & Post & Prä & Post \\
\hline Belastung $\mathrm{at}_{\mathrm{t}}(\mathrm{W})$ & $\pm 22,4$ & $57,5 \pm 23^{*}$ & $52,2 \pm 23,3$ & $46,1 \pm 32,9$ \\
\hline Belastung $_{\max }(\mathrm{W})$ & $\pm 39,1$ & $93 \pm 43^{* *}$ & $89,4 \pm 41,1$ & $86,1 \pm 46,2$ \\
\hline $\mathrm{Hf}_{\mathrm{at}}(\mathrm{spm})$ & $110,9 \pm 12,4$ & $120,9 \pm 18,4^{*}$ & $116,0 \pm 4,3$ & $114,8 \pm 4$ \\
\hline $\mathrm{Hf}_{\max }(\mathrm{spm})$ & $134,2 \pm 16,4$ & $140,2 \pm 18,8^{*}$ & $133,4 \pm 12,2$ & $132,7 \pm 10,5$ \\
\hline$\Delta \mathrm{Hf}_{30 \mathrm{sec}}(\mathrm{spm})$ & $5 \pm 3,9$ & $7,2 \pm 5^{*}$ & $3,3 \pm \quad 3,5$ & $2,8 \pm \quad 2,7$ \\
\hline $\mathrm{VOZ}_{\mathrm{at}}(\mathrm{L} / \mathrm{min})$ & $945,7 \pm 281,3$ & $1079,5 \pm 260,8^{*}$ & $973,3 \pm 266,6$ & $876,8 \pm 285,3^{* *}$ \\
\hline $\mathrm{VOZ}_{\text {peak }}(\mathrm{L} / \mathrm{min})$ & $1302,9 \pm 413,3$ & $1437,6 \pm 381,1^{*}$ & $1435,3 \pm 537,6$ & $1365,1 \pm 541,4$ \\
\hline $\mathrm{VE}_{\max }(\mathrm{L} / \mathrm{min})$ & $46 \pm 14,3$ & $50,2 \pm 14,9^{*}$ & $48,7 \pm 12,9$ & $47,8 \pm 12,3$ \\
\hline $\begin{array}{l}\text { Laktat }_{4 \min } \\
(\mathrm{mmol} / \mathrm{L})\end{array}$ & $2,38 \pm 0,61$ & $2,01 \pm \quad 0,7^{*}$ & $2,2 \pm \quad 0,7$ & $2,51 \pm 1,13$ \\
\hline \multicolumn{5}{|c|}{ 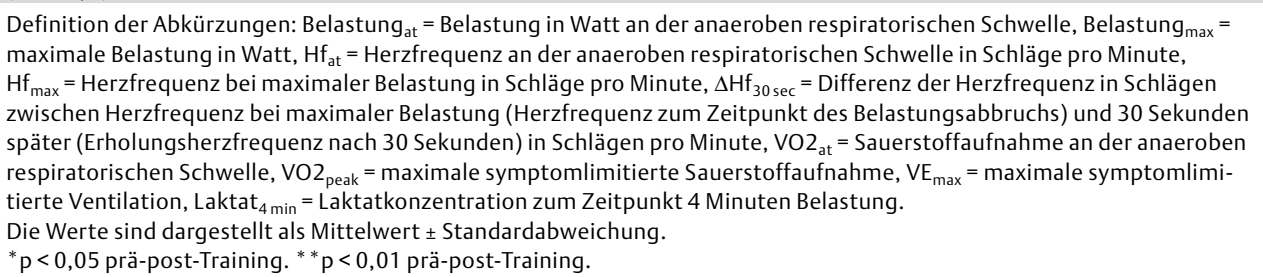 } \\
\hline
\end{tabular}

drücke für Sauerstoff $\left(\mathrm{PaO}_{2}\right)$ and Kohlendioxid $\left(\mathrm{PaCO}_{2}\right)$ (Master LAB AVL Compact 2, AVL, Graz, Austria) zu bestimmen.

\section{Sechs-Minuten-Gehstrecke}

Der Sechs-Minuten-Gehstrecken-Test wurde an zwei unterschiedlichen Tagen durchgeführt. Die Länge des Flurs betrug 80 Meter.

\section{Krafttests}

Die Handkraft wurde mit einem Handkraftdynamometer (JAMAR, J.A. Preston Corporation, Jackson, MI), die maximale isometrische Kraft des m. quadrizeps femoris mit einer Drehmomentmesseinrichtung (m3 Diagnos +, Fa. Schnell, Peutenhausen, Germany) ermittelt.

\section{Lungenfunktion}

Die Lungenfunktion wurde in einem Bodyplethysmographen (Jaeger, Würzburg, Germany) durchgeführt. Zusätzlich zu Standardparametern wurde die Diffusionskapazität mittels singlebreath-method bestimmt.

\section{Arterielle Blutgase}

Die arteriellen Blutgase (Ohrläppchen) wurden durch die Mikromethode (Master LAB AVL Compact 2, AVL, Graz, Austria) ermittelt.

\section{Körperkonstitution}

Die Körperkonstitution wurde durch ein bioelektrisches Impendanzmessgerät erhoben (TBF 300, Tanita, Sindelfingen, Germany).

\section{Lebensqualität}

Für die generische Lebensqualität wurde der SF36 [6] herangezogen, der auch für Patienten mit COPD [7] evaluiert ist. Die krankheitsspezifische Lebensqualität wurde durch den Saint George Respiratory Questionnaire ermittelt [8].

\section{Statistik}

Es erfolgt ein Inter- und Intragruppenvergleich. Aufgrund der Stichprobengröße und der Verteilungseigenschaften werden nonparametrische Verfahren eingesetzt, zum Ausgleich der Gruppenunterschiede ein sog. Solomonplan [9]: Es erfolgt eine Differenzbildung von Abschluss- und Eingangstestwerten, es verbleiben reine Veränderungsraten als Grundlage der Berechnungen für den Vergleich zweier unabhängiger Stichproben. Als statistisches Verfahren liegt der U-Test von Mann-Whitney zugrunde. Für den Intragruppenvergleich ist der Vorzeichenrangtest von Wilcoxon indiziert. Als Signifikanzniveau wurde $\alpha=0,05$ gesetzt. Die Ergebnisse werden als Mittelwert \pm Standardabweichung im Text und in den Abbildungen präsentiert.

\section{Ergebnisse}

Effekte des Trainingsprogramms auf die Belastbarkeit In der Trainingsgruppe fand sich eine Rechtsverschiebung der respiratorischen anaeroben Schwelle ( Tab.5). Die Wattzahl stieg um von $47 \pm 22$ Watt auf $57,5 \pm 23$ Watt (22,5\%) signifikant $(p=0,02)$ an. Die Herzfrequenz erhöhte sich signifikant $(p=0,03)$ von $110,9 \pm 12 \mathrm{spm}$ auf $120,9 \pm 18 \mathrm{spm}(9 \%)$. Es kam zu einer signifikanten Erhöhung $(\mathrm{p}=0,03)$ der Sauerstoffaufnahme von $0,95 \pm 0,28 \mathrm{~L} / \mathrm{min}$ auf $1,08 \pm 0,26 \mathrm{~L} / \mathrm{min}(14 \%)$. In der Kontrollgruppe sank die Wattzahl an der anaeroben respiratorischen Schwelle um 6 Watt nicht signifikant ab. Die Herzfrequenz blieb nahezu unverändert, während die Sauerstoffaufnahme an der anaeroben respiratorischen Schwelle eine signifikanten Erniedrigung $(\mathrm{p}=0,006)$ aufwies.

Auch die maximale Belastbarkeit stieg in der Trainingsgruppe an. Die Wattzahl erhöhte sich signifikant $(\mathrm{p}=0,007)$ von $82 \pm 39$ Watt auf $93 \pm 43$ Watt (14\%). Die maximale Herzfrequenz stieg signifikant $(p=0,02)$ von $134,2 \pm 16$ Schlägen pro Minute auf $140,2 \pm 19$ Schläge pro Minute (5\%) an. Es kam zu einer signifikanten Erhöhung $(p=0,01)$ der symptomlimitierten maximalen Sauerstoffaufnahme von $1,30 \pm 0,41 \mathrm{l} / \mathrm{min}$ auf $1,44 \pm 0,38 \mathrm{l} / \mathrm{min}$ (10\%). Die maximale Ventilation stieg signifikant $(p=0,03)$ von $46 \pm 14 \mathrm{l} / \mathrm{min}$ auf $50 \pm 15 \mathrm{l} / \mathrm{min}$ (9\%). In der Kontrollgruppe fanden sich tendenzielle, aber nicht signifikante Verschlechterun- 


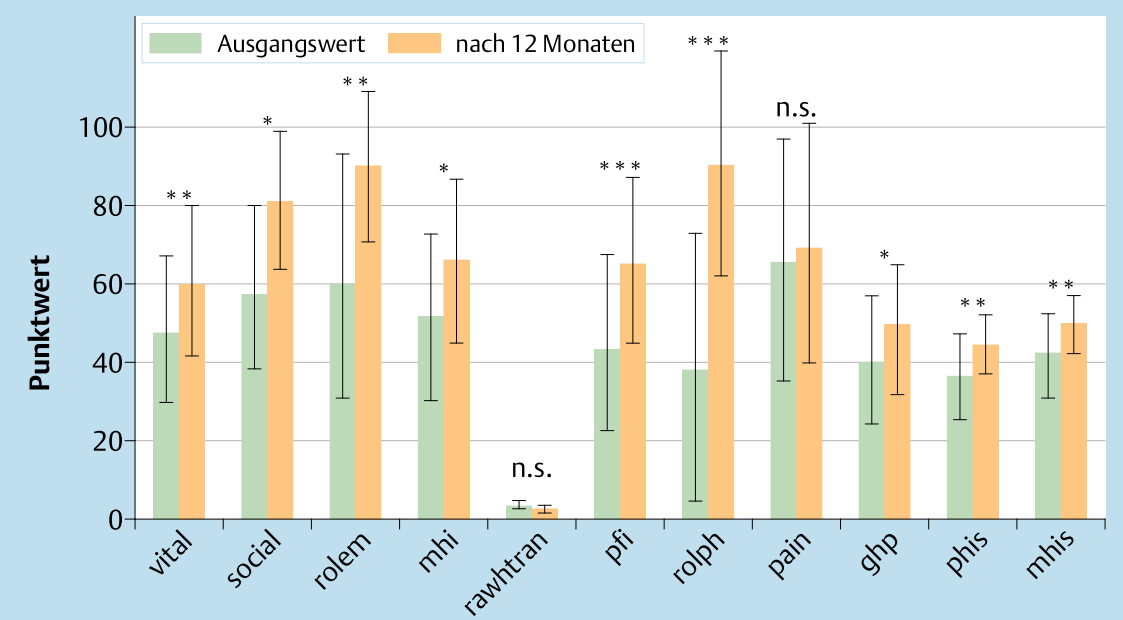

Abb. 2 SF36: Dimensionen und Subskalen in der Trainingsgruppe

Definition der Abkürzungen: vital = Vitalität, social = Soziale Funktionsfähigkeit, rolem = Emotionale Rollenfunktion, $\mathrm{mhi}=$ Psychisches Wohlbefinden, rawhtran = Veränderung der Gesundheit, pfi = Körperliche Funktionstüchtigkeit, rolph = Körperliche Rollenfunktion, pain = Köperliche Schmerzen, ghp = Allgemeine Gesundheitswahrnehmung, phis = Psychische Summenskala, mhis $=$ Körperliche Summenskala. Die Werte sind als Mittelwert \pm Standardabweichung dargestellt.

n. $\mathrm{s} .=$ nicht signifikant

${ }^{*} \mathrm{p}<0,05$ prä-post-Training

${ }^{* *} p<0,01$ prä-post-Training

${ }^{* * *} \mathrm{p}<0,001$ prä-post-Training.

SF-36: Dimensionen und Subskalen

gen. Vergleicht man die Werte zum Zeitpunkt der maximalen Belastung im Eingangstest mit den Werten bei vergleichbarer Belastungsstufe im Abschlusstest, also Wattzahl zum Zeitpunkt des Belastungsabbruchs im ET [10], waren im Abschlusstest die Sauerstoffaufnahme um 5\%, die Kohlendioxidabgabe um $8 \%$, die Herzfrequenz um 5\% und die Ventilation um 6\% niedriger. Der Abfall der Herzfrequenz nach maximaler Belastung im Zeitraum 30 Sekunden und 2 Minuten hat sich signifikant erhöht ( $p=0,02$ und $p=0,03$ ), die Erholungsfähigkeit hat sich verbessert. In der Kontrollgruppe fanden sich keine Veränderungen. Die Laktatproduktion bei vergleichbaren Zeitpunkten (2, 4, 6, 8 Minuten) ist signifikant geringer $(p=0,003, p=0,01, p=0,02, p=0,02)$. Die Kontrollgruppe weist tendenzielle jedoch nicht signifikante Verschlechterungen auf. Abschließend eine tabellarische Darstellung verschiedener Parameter der Ergospirometrie.

\section{Effekte des Trainingsprogramms auf die Sechs-Minu- ten-Gehstrecke}

Die Ergebnisse der 6 MWD sind sowohl im Intra- als auch Intergruppenvergleich auf dem $1 \%$-Niveau signifikant. Die Verbesserung in der Trainingsgruppe von 414,7 $\pm 89,7 \mathrm{~m}$ auf 494 \pm 75,9 $\mathrm{m}$ beträgt absolut 79 Meter (16\%) und ist damit auch klinisch relevant [11]. Die Kontrollgruppe weist keine signifikanten Veränderungen auf.

\section{Effekte des Trainingsprogramms auf die Handkraft}

In der Trainingsgruppe stieg die Handkraft links von $28 \pm 14,4 \mathrm{~kg}$ auf $37,5 \pm 11,9 \mathrm{~kg}(30 \%)$ und rechts von $30,3 \pm 14 \mathrm{~kg}$ auf $39,3 \pm$

$12,2 \mathrm{~kg}(34 \%)$ jeweils signifikant $(\mathrm{p}=0,003)$ an. Bei der Beinkraft konnte eine signifikante Steigerung $(p=0,02)$ von 196,7 \pm $111,7 \mathrm{Nm}$ auf $234 \pm 105,2 \mathrm{Nm}$ (19\%) festgestellt werden. Die Kontrollgruppe weist bei allen Parametern keine signifikanten Veränderungen auf.

\section{Effekte des Trainingsprogramms auf Körperkonstitution und Lungenfunktion}

Körpergewicht und Körperkonstitution wurden nicht signifikant in beiden Gruppen verändert. Alle Lungenfunktionsparameter wiesen weder in der Trainingsgruppe noch in der Kontrollgruppe signifikante Veränderungen auf.
Effekte des Trainingsprogramms auf die Lebensqualität Im SF36 fanden sich in der Trainingsgruppe signifikante Verbesserungen verschiedener Subscores sowie der psychischen Summenskala $(p=0,01)$ und der körperlichen Summenskala $(p=0,007)$. Vergleiche hierzu $\bigcirc$ Abb. 2. In der Kontrollgruppe kam es zu einer signifikanten Verschlechterung der Subscores Vitalität (vital) und Rollenfunktion (rolem). Alle anderen Subscores ebenso wie die Summenskalen blieben vergleichbar.

Im Saint George Respiratory Questionnaire kam es zu einer signifikanten Verbesserung der Domänen Einschränkungen und Aktivitäten sowie des Gesamtscores $(\mathrm{p}=0,02)$. Die absolute Verbesserung des Gesamtscores übersteigt 4 Punkte und ist damit als klinisch relevant einzustufen. Wie in $\mathbf{A b b} \mathbf{3}$ zu erkennen, weist die Kontrollgruppe lediglich tendenzielle Veränderungen auf.

\section{Diskussion}

\section{Anmerkungen zur Methodik}

Da bei einer möglichen Breitenanwendung in den Rahmenvereinbarungen über den Rehabilitationssport (SGB XI, §44) eine Obergrenze von 15 Teilnehmern pro Gruppe empfohlen wird, haben wir in dieser Pilotstudie eine entsprechende Stichprobenzahl eingeschlossen.

Die Ausfallquote der an dieser Studie teilnehmenden Patienten war relativ hoch, die Gründen hierfür eher atypisch: Die infektbedingte Anzahl der Ausfälle war geringer als die aufgrund anderer Erkrankungen oder Verletzungen. Auffällig war das gehäufte Auftreten der peripheren arteriellen Verschlusskrankheit (paVK), welche sich bei den Teilnehmern, überwiegend Raucher oder Exraucher, mit zunehmendem Trainingszustand demaskierte. Für künftige Interventionen ist eine paVK ggf. in die Ausschlusskriterien miteinzubeziehen. Aufgrund der Stichprobengröße und den Verteilungseigenschaften wurden ein Solomonplan gewählt und nonparametrische Verfahren eingesetzt. Trotz des randomisierten kontrollierten Designs ist die statistische Aussagekraft der Studie deshalb limitiert.

\section{Interpretation der Daten}

Durch die Herzfrequenzaufzeichnungen beim Krafttraining konnte bei den häufig dekonditionierten Patienten festgestellt werden, dass ein Training an den verschiedenen Krafttrainings- 


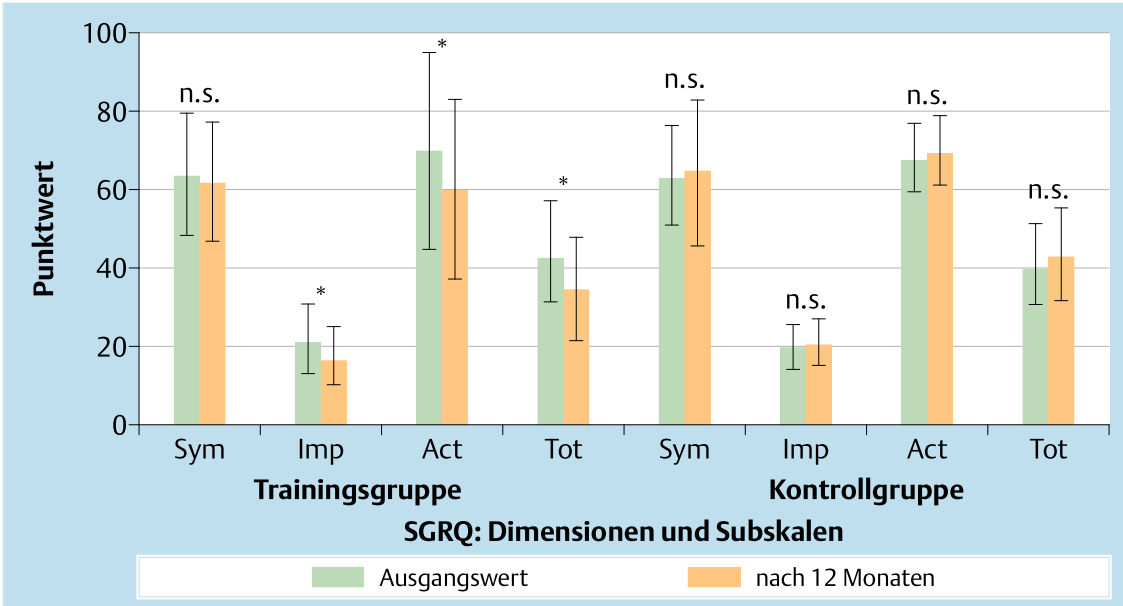

Abb. 3 Ergebnisse des Saint George Respiratory Questionnaire

Definition der Abkürzungen:

Sym = Symptome, Imp = Auswirkungen,

Act $=$ Aktivität, Tot $=$ Gesamtscore

Die Werte sind als Mittelwerte \pm Standard-

abweichung dargestellt.

n. s. = nicht signifikant

${ }^{*} \mathrm{p}<0,05$ prä-post-Training.

Ausgangswert

Monaten

geräten einen Herz-Kreislauf-wirksamen Reiz darstellt. Krafttraining und der Übergang des Trainings in den Ausdauerbereich erscheinen geeignet, COPD-Patienten auf intensive und längere Ausdauerbelastungen vorzubereiten. Die von uns praktizierte Vorgehensweise, über eine Lernphase am geführten Krafttrainingsgerät ein Freihanteltraining und Übungen mit dem Theraband in den Kurseinheiten vorzubereiten, erwies sich als gut. Ungelöst ist das Problem, ein intensives Krafttraining in einer Lungensportgruppe umzusetzen, wo in der Regel nur Therabänder oder Hanteln zur Verfügung stehen.

In dieser Studie wurde ein normaler Trainingsaufbau, Grundlagentraining (Makrozyklus „Ausdauer: Aerobes Training“) vor anaerobem Training (Makrozyklus „Ausdauer: anaerobes Training“), gewählt. Die eingesetzte Trainingskonzeption führte gegenüber (teil)stationären Trainingsprogrammen zu vergleichbaren Ergebnissen von Casaburi u. Mitarb. 1991, 1997 [10,13] und Maltais u. Mitarb. 1996, 1997 [14,15], so dass die Frage nach einer optimalen Konzeption im Bereich des Ausdauertrainings nicht abschließend beantwortet werden kann. Eine Optimierung der Trainingseffekte durch Modifikationen für Phase IV des hochintensiven Trainings (keine Kurzzeitintervalle), aber auch Veränderungen des Grundlagentrainings in Phase III (höhere Umfänge mit niedrigeren Intensitäten über längere Zeiträume) erscheinen möglich.

Die durchschnittliche Trainingszeit pro Person von ca. 166 Stunden pro Jahr entspricht bei Einordnung der Belastung in Belastungsumfänge pro Jahr [16] der Kategorie „Anfänger“, die durchschnittlich weniger als 200 Stunden pro Jahr für das Training aufwenden. Zum Vergleich trainieren Leistungssportler 300 - 400 Stunden/Jahr. Unter diesem Aspekt erscheint es sinnvoll, die Patienten im mehrjährigen Trainingsprozess auf mindestens ca. 200 Stunden/Jahr Training und körperliche Aktivität einzustellen.

Diese Arbeit ist als Pilotstudie zu werten. Die erzielten Effekte sind auf das Trainingsprogramm zurückzuführen. Als Nachweis der Effektivität von „Lungensport“ allgemein, zumindest nach aktuellem Verständnis (Training $1 \times$ pro Woche), ist diese Studie nur bedingt anzuführen, da die Intervention deutlich höhere Trainingsumfänge hatte. Für die Effekte, gerade im Bereich Lebensqualität, spielen die regelmäßigen Treffen der Teilnehmer gruppendynamische Prozesse im Sinne eines psychosozialen Supports - sicherlich eine Rolle.

\section{Konsequenzen für das Design ambulanter}

\section{Trainingsprogramme für Patienten mit COPD}

Die Leistungsfähigkeit von COPD-Patienten aller Schweregrade lässt sich innerhalb von mehreren Wochen verbessern, der Trainingseffekt geht jedoch ohne entsprechende Weiterführung des körperlichen Trainings wieder verloren.

Um Trainingseffekte von COPD-Patienten dauerhaft zu erhalten, ist ein flächendeckendes und wohnortnahes Trainingsangebot zu fordern. Für ein ambulantes Konzept der nichtmedikamentösen Therapie der COPD müssen bewährte Inhalte (z. B. körperliches Training, Patientenschulung, Physiotherapie) und ihre Umsetzungsmöglichkeiten analysiert werden. Es gilt u.a. die räumlichen (z.B. Turnhalle), gerätemäßigen (z.B. Krafttrainingsgeräte, Pulsoximeter, Fahrradergometer) und personellen (z.B. Therapeut, Fachkraft) Voraussetzungen zur Umsetzung nachgewiesen effektiver Maßnahmen festzulegen. In Abhängigkeit von den individuellen Gegebenheiten wie etwa Schweregrad der Erkrankung, Komorbidität, Motivation, Mobilität, etc. könnten dann verschiedene Inhalte kombiniert, unter Ausschöpfung der wohnortnahen Gegebenheiten (Physiotherapiepraxen, niedergelassene Ärzte, Vereine, Fitnessstudio, Heimtraining, etc.) umgesetzt werden („Settingmix“). Voraussetzung für diese Vorgehensweise ist die Erarbeitung eines schweregradabhängigen koordinativen und konditionellen „Anforderungsprofils“. Zwar werden verschiedene Parameter durch Assessmentverfahren wie die 6-Minuten-Gehstrecke, Spiroergometrie etc. erhoben, es ist jedoch noch kein detailliertes Trainingsprofil in Abhängigkeit von Schweregrad und limitierendem Faktor definiert. Anzustreben ist die Einrichtung einer „multidisziplinären“ Arbeitsgruppe (Sport- und Physiotherapeuten, Ärzte etc.) welche neben diesem Anforderungsprofil konkrete schriftliche Ausarbeitungen im Bereich Methodik und Didaktik (Gruppenanwendung mit ca. 15 Personen) für die einzelnen Bereiche (u.a. physiotherapeutische Techniken, Ausdauertraining und Symptomsensibilisierung etc.) mit dem Ziel der standardisierten Umsetzung durch eine Person erarbeitet.

Durch die in der Studie vorgeschlagene Terminologie und Systematik der Trainingsinhalte wurde eine Voraussetzung zur Erstellung solcher Anforderungsprofile geschaffen. Die gewählte Periodisierung des Trainings (Aufteilung des Trainings in mehrere Makrozyklen) bringt Transparenz der Trainingsinhalte. Eine Weiterführung dieser Aufarbeitung in Form von Rahmentrainingsplänen und oder exakte Vorgaben für die Ausgestaltung einzelner Zyklen ist daher möglich. Auch Sondersituationen wie 
Aufnahme von Neueinsteigern oder Teilnahme nach Infekt könnten durch zyklisiertes Vorgehen letztlich leichter (wieder) durchgeführt werden.

Das vorliegende Trainingskonzept ist für die COPD bislang noch nicht in der Literatur erwähnt worden. Die effektiven Komponenten der pneumologischen Rehabilitation werden nach dem Prinzip eines Baukastensystems kombiniert. Hervorzuheben ist die zeitliche Gliederung in einzelne Makrozyklen entsprechend dem jahreszeitlichen Verlauf - Schwerpunkte Theorie, Koordination und Beweglichkeit in der Übergangsphase Herbst-Winter, Kraft in den Wintermonaten und Ausdauer in den stabilen Sommermonaten - und die Umsetzung in verschiedenen „Settings“ (Lungensportgruppe, Heimtraining, Gesundheits- und Fitnesseinrichtung). Durch einen fließenden Übergang in den vier Phasen (Makrozyklen) und den Wechsel von Trainingsinhalten, -mittel, -methoden und -umfänge werden bestehende Defizite systematisch angegangen. Mit geringfügigen Modifikationen kann diese Konzeption von einem Übungsleiter alleine durchgeführt werden und ist somit für eine Breitenanwendung geeignet.

\section{Anmerkung}

Diese Studie wurde ermöglicht durch die freundliche Unterstützung der Fa. ZAMBON GmbH und dem Universitätsbund der Universität Erlangen-Nürnberg

\section{Literatur}

1 Lacasse Y, Brosseau L, Milne S et al. Pulmonary rehabilitation for chronic obstructive pulmonary disease. Cochrane Database Syst Rev 2002; (3) $\mathrm{CD} 003793$

2 Ries A, Kaplan R, Limberg T et al. Effects of pulmonary rehabilitation on physiologic and psychosocial outcomes in patients with chronic obstructive pulmonary disease. Ann Intern Med 1995; 122: 823-832
3 Rochester $C$. Exercise training in chronic obstructive pulmonary disease. J Rehabil Res Dev 2003; 40: 59-80

4 World Health Organization. The GOLD global strategy for the management and prevention of COPD. www.goldcopd.com

5 Wassermann K, Hansen J, Sue S et al. Principles of Exercise Testing and Interpretation (3rd ed.). Lippincott: Williams \& Wilkins, 1999

6 Ware J, Sherbourne C. The MOS 36-item short-form health survey (SF-36). Conceptual framework and item selection. Med Care 1992; 30: $473-483$

7 Mahler D, Mackowiak J. Evaluation of the short-form 36-item questionnaire to measure health-related quality of life in patients with COPD. Chest 1995; 107: 1585-1589

8 Jones P, Quirk F, Baveystock C et al. A self-complete measure of health status for chronic airflow limitation. The St. George's Respiratory Questionnaire. Am Rev Respir Dis 1992; 145: 1321 - 1327

9 Bortz J, Lienert G. Kurzgefasste Statistik für die klinische Forschung: ein praktischer Leitfaden für die Analyse kleiner Stichproben. BerlinHeidelberg: Springer, 1998

10 Casaburi R, Patessio A, Ioli F et al. Reductions in exercise lactic acidosis and ventilation as a result of exercise training in patients with obstructive lung disease. Am Rev Respir Dis 1991; 143: 9- 18

11 Elpern E, Stevens D, Kesten S. Variability in performance of timed walk tests in pulmonary rehabilitation programs. Chest 2000; 118: $98-105$

12 O'Shea S, Taylor N, Paratz J. Peripheral Muscle Strength Training in COPD. Chest 2004; 126: $903-904$

13 Casaburi R, Porszasz J, Burns $M$ et al. Physiologic benefits of exercise training in rehabilitation of patients with severe chronic obstructive pulmonary disease. Am J Respir Crit Care Med 1997; 155: 1541 - 1551

14 Maltais F, Le BP, Simard C et al. Skeletal muscle adaptation to endurance training in patients with chronic obstructive pulmonary disease. Am J Respir Crit Care Med 1996; 154: $442-447$

15 Maltais F, Le BP, Jobin J et al. Intensity of training and physiologic adaptation in patients with chronic obstructive pulmonary disease. Am J Respir Crit Care Med 1997; 155: 555- 561

16 Zintl F, Eisenhut A. Ausdauertraining. Grundlagen - Methoden - Trainingssteuerung. München-Wien-Zürich: BLV Verlagsgesellschaft $\mathrm{mbH}, 2001$

17 Murray C, Lopez A. Alternative projections of mortality and disability by cause 1990 - 2020: Global burden of Disease Study. Lancet 1997; 349: $1498-1504$ 